\title{
Cultural Adaptation and Examination of Metric Characteristics Shirom-Melamed Burnout Questionnaire (SMBQ) On a Sample of Pharmacists in Serbia
}

\author{
Dragana Jocic ${ }^{1 *}$, Nela Djonovic ${ }^{2}$, Dusanka Krajnovic ${ }^{3}$, Srdjan Stefanovic ${ }^{4}$, Svetlana Stojkov ${ }^{5}$, Sanja \\ Kocic $^{6}$ \\ 1BENU Pharmacy, Kumodraska 239, 11000 Belgrade, SERBIA. \\ ${ }^{2}$ University of Kragujevac - Faculty of Medical Sciences, Department of Hygiene and ecology, Svetozara Markovića 69, Kragujevac, \\ SERBIA. \\ ${ }^{3}$ University of Belgrade - Faculty of Pharmacy, Department of Social Pharmacy and Pharmaceutical Legislation, Vojvode Stepe 450, \\ Belgrade, SERBIA. \\ ${ }^{4}$ University of Kragujevac - Faculty of Medical Sciences, Department of Pharmacy, Svetozara Markovića 69, Kragujevac, SERBIA. \\ ${ }^{5}$ Pharmaceutical Chamber of Serbia, Mutapova 25, Belgrade, SERBIA. \\ ${ }^{6}$ University of Kragujevac - Faculty of Medical Sciences, Department of social medicine, Svetozara Markovića 69, Kragujevac, SERBIA.
}

\begin{abstract}
Introduction: Due to the nature of work in pharmacies pharmacists may occur job burnout, which can affect the quality of health services. It is necessary to use validated instruments to test the burnout. One type of instrument is Shirom-Melamed Burnout Questionnaire (SMBO). Objective: The primary objectives of this work are: cultural adaptation and assessment metric characteristics of SMBO in a population of pharmacists in Serbia. Alternative objective is evaluate the degree of burnout in the pharmacist population. Methods: For comparison Maslach Burnout Inventory (MBI) was used. All licensed pharmacists have received an email by the Pharmaceutical Chamber of Serbia, with a link for accessing the questionnaires. The questionnaires were completed by 514 pharmacists. Results and Discussion: Professional translators carried out the translation using translation steps like forward translation and backward translation. Were calculated following metric characteristics: $\alpha$ - reliability coefficient Cronbach and associates; $\beta$ - the reliability of the first principal component; $\psi 1$ - representativeness; h1 - the homogeneity (the average correlation of scale items), extent of curvature, horizontal (Skewness) and vertical (Kurtosis). Correlations between subscales are examined. Latent structure SMBO (maximumlikelihud extraction) is examined. To select a number of factors, the method of parallel analysis was used. For calculation number of latent factors the Hull method was applied. Concurrent validity was examined by correlating the results of the questionnaire SMBO and MBI. To confirm a one factor solution of the scale, factor analysis was used. Conclusion: All methods show that it is best to keep only one factor. SMBO satisfies theoretical statistical criteria of reliability and validity and can be used to estimate job burnout at pharmacist population.
\end{abstract}

Key words: Shirom-Melamed Burnout Questionnaire, Cultural Adaptation, Metric Characteristics. Job Burnout, Pharmacists, Serbia.
Submission Date: 02-06-2017; Revision Date: 18-08-2017; Accepted Date: 10-01-2018

DOI: 10.5530/ijper.52.1.19 Correspondence: Dragana Jocic, BENU Pharmacy, Kumodraska 239, 11000 Belgrade, SERBIA. Phone no: +381 63414025 Email Id: jocicdragana@ yahoo.com

\section{INTRODUCTION}

Research conducted at a sample of pharmacists in primary health care in Serbia, shows that almost half of the surveyed pharmacists were at burnout risk. The greatest burnout risk had pharmacists from the territory of
Belgrade and older pharmacists with more years of experience. ${ }^{1,2}$

Burnout is a mental condition defined as a result of continuous and long-term stress exposure, particularly related to psychosocial

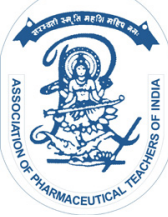

www.ijper.org 
factors at work. ${ }^{3}$ Burnout syndrome is a specific syndrome, which is caused by prolonged exposure to work stress, and is occurs primarily in occupations characterized by working with people in emotionally demanding situations. Maslach and Jackson give a definition that the burning syndrome is emotional exhaustion, depersonalization, and reduced personal accomplishment that occurs in individuals who work with people. ${ }^{4-7}$ Burnout does not develop with each person who is chronically under stress. Stress is a personal experience, and therefore there is no generalized boundaries where positive stress becomes negative. If different types of stress is multiply or stress is increased, the individual reaches his personal limit. The optimal level of stress can have influence to person to behave a more productive, more creative and to increase the working enthusiasm. However, if the stress levels too high because of the job demands and pressures, working enthusiasm will be decrease. Too much stress will reduce a person's health and her mental stability. ${ }^{5-7}$

It is essential to recognize when the workload exceeds the individual limit of tolerance of stress, and when the requirements of the workplace and the environment overcome individual abilities, desires and expectations that are necessary in order to respond to these requests. ${ }^{8}$ To test the psychological phenomenon of the healthy population, it is necessary to use validated instruments. A large number of instruments that are used to assess the burnout risk are on the market. Many of these instruments relate to factors that are not exclusive in the field work activities. It is very important that there is an instrument that will be used to assess job burnout, which will be focused on the domains of work activities, but wich will cover many of phisical ans psycological simptoms of burnout.

One such instrument is Shirom-Melamed Burnout Questionnaire (SMBQ).

The primary objectives of this work are:

The cultural adaptation and metric characteristics assessment of SMBQ as an instrument for examine burnout in a population of pharmacists in Serbia.

Alternative objective is evaluate the degree of burnout in the pharmacist population.

\section{MATERIALS AND METHODS Study Design and Ethical Approval}

Before the start of the research, the consent of the author for the use of questionnaires was obtained.

The Ethics Committee of the Pharmaceutical Chamber of Serbia approved the study (Approval of the Ethics
Committee of the Pharmaceutical Chamber of Serbia, No 404/1-4, 6/5/2016).

All participants received a full explanation of the study and were guaranteed anonymity. Informed consent was obtained by all participants included in the study. No financial compensation was given to any participant.

A cross-sectional survey design was used. Data were obtained from self-administered instrument from January 2017 to March 2017.

\section{Study Criteria}

The target population in the study were licensed pharmacists employed in pharmacies at the primary level of health care in Serbia. At the start of the study are precisely defined inclusion criteria and exclusion criteria for the study. Based on these criteria eliminated the persons who do not meet the criteria.

Inclusion criteria: adults with a degree from the Faculty of Pharmacy, licensed pharmacists employed in pharmacies at the primary health care level in Serbia, people who voluntarily agree to participate in the study.

Exclusion criteria: pharmacists without a license, interns, persons employed in a pharmacy that do not have completed the Faculty of Pharmacy, people who refuse to participate in the study, or if there is any objective reason that prevents or hinders participation in the study.

\section{Sample}

The sample size was calculated by the statistical data processing.

Calculation of the total sample is based on the results of the studies previously published of similar design and a known size of the population (6124 licensed pharmacist). Based of the $80 \%$ strenth of study, a known size of the population, of the probability of the first type error $(\alpha)$ that is 0.05 , an effect size that is 0.5 , it can be calculated that in the study least 362 subjects will be included.

List of all licensed pharmacists on the territory of Serbia was obtained by the Pharmacists' Association of Serbia. There were 6124 licensed pharmacists on the territory of Serbia at the time of the survey was conducted. By the Pharmacists' Association of Serbia all licensed pharmacists have received an email with a link for accessing the questionnaires. The questionnaires were completed by 514 pharmacists.

\section{Research instruments}

To assess the burnout risk Shirom-Melamed Burnout Questionnaire (SMBQ) was used.

For validation SMBQ Maslach Burnout Inventory (MBI) was used. 
The SMBQ instrument originally designed to measure burnout as a multidimensional construct consisting of emotional exhaustion, physical fatigue, and cognitive weariness, which together represents the core component of burnout. Later, constructs related to tension and listlessness are added. ${ }^{3}$

Shirom-Melamed Burnout Questionnaire is a seven-point Likert scale and is designed for measuring job burnout. It have 22 items which consists of the following sub-scales: Emotional exhaustion (EE), Physical fatigue (PF), Cognitive weariness (Cog), Tension (TE) and Listlessness (LIS). ${ }^{3}$

Each item is rated using a seven-point scale ranging from 1 "Never or almost never" to 7 "Always or almost always".

For each sub-domain, and the entire scale, the total score is averaged by dividing by the number of items in the domain. A total score in the range of 22 to 154 is converted into the average results for the sets to 4 .

Average score of 0 to 3.0 means that there is not risk of job burnout, the average score of 3.1 to 3.6 is normal under optimal stress at work, while the average results from 3.7 to 4.0 means high level of burnout when exist the high risk of job burnout. ${ }^{8}$

In this paper, this instrument was used to estimate burnout. Burnout at work cannot be separated from burnout in everyday life, and this scale covers a wide range of somatic and psychological aspects of burnout.

The most widely used instrument is the Maslach Burnout Inventory (MBI) and the conceptual basis for MBI is thus often considered as synonymous with the construct burnout. Maslach and colleagues originally defined burnout as a psychological syndrome of emotional exhaustion, depersonalization (later replaced with the construct cynicism) and reduced effectiveness or personal accomplishment, which makes this scale a multidimensional construct. The original MBI was developed during the late 1970s, based on a program of field research within healthcare and the human services. The labels for the three dimensions reflected the focus on occupations where workers interacted extensively with other people. ${ }^{7}$

MBI is the most commonly used tool to self-assess whether you might be at risk of burnout. To determine the risk of burnout, the MBI explores three components or thre section: exhaustion, depersonalization and personal achievement. ${ }^{7}$

Section A: Exhaustion - Testifies to fatigue at the very idea of work, chronic fatigue, trouble sleeping, physical problems.

- Total 17 or less: Low-level burnout
- Total between 18 and 29 inclusive: Moderate burnout

- Total over 30: High-level burnout

Section B: Depersonalization (or loss of empathy). The notion of detachment is excessive, leading to cynicism with negative attitudes with regard to patients or colleagues, feeling of guilt, avoidance of social contacts and withdrawing into oneself.

- Total 5 or less: Low-level burnout

- Total between 6 and 11 inclusive: Moderate burnout

- Total of 12 and greater: High-level burnout

Section C: Personal Achievement - The reduction of personal achievement: The individual assesses himself negatively, feels he is unable to move the situation forward. This component represents the demotivating effects of a difficult, repetitive situation leading to failure despite efforts.

- Total 33 or less: High-level burnout

- Total between 34 and 39 inclusive: Moderate burnout

- Total greater than 40: Low-level burnout.

\section{Translation and cultural adaptation of the questionnaire}

There are a number of general and specific standardized questionnaire in the scientific literature, but most of them are created in English. Therefore, at the beginning of the preparation of the research necessary to adjust questionnaire language translation to the language on which conducts research and of the population respondents language. There are several published guidelines for translation and cultural adaptation of the questionnaire..$^{-11}$ One often used guideline is the guideline of the International Society for Pharmacoeconomics and Outcomes Research - ISPOR. ${ }^{10}$ According to ISPOR guidelines, translating and adapting questionnaires process consist several steps: preparation (definition of the concept of research and getting a license to use the questionnaire), translating "advance" (two independent translators translate questionnaire from the mother language to the language used in conducting research), harmonization translated version and the original version, the translation of "backward" (from the language in which to conduct research to the home language survey), harmonization of all translated versions, cognitive testing (testing of questionnaires on the population until 10 participants in order to test interpretation, clarity and understanding of the issue), entering corrections, control questionnaires reading and writing the final report. ${ }^{10}$

Other published guidelines are variations of ISPOR guidelines, and differ in the number and type of translators 
(in steps of translating "forward" and "backward"), as well as in the number of respondents for the process of cultural adaptation of the questionnaire. ${ }^{10-12,13}$

\section{Cultural adaptation of the questionnaire}

The cultural adaptation of the SMBQ questionnaire was made according to ISPOR guidelines because this approach use holistic perspective that synthesized the full spectrum of published methods. This approach to translation and cultural adaptation was considered systematically in terms of rationale, components, key actors, and the potential benefits and risks associated with each approach and step. The only deviation in relation to ISPOR guidelines refers to the number of respondents in the prevalidation study. The ISPOR guidelines recommend that a prevalidation study be conducted on a sample of 10 subjects. We used a sample of 30 respondents.

First step was translation and cultural adaptation of the questionnaire. The translation and cultural adaptation of the questionnaire were consisted of several stages: translation, synthesis, reverse translation, review by an expert team, testing and collection of written reports.

Next steps were conducted:

- Obtaining licenses for the use of questionnaires

- Translation of the SMBQ from the original language of the questionnaire to Serbian language (translation in advance). This step was implemented by two translators whose native language is Serbian and who are familiar with the concept of research - the first step in the adaptation of the translation from the source language to the target language (Serbian). Translation service requires two independent translator (TRANSLATOR T1 and T2), whose native language is Serbian and a second language of the source language of the questionnaire. Both translators provided written translation of the report, which they did.

- Unified version was formed - Connected these two translations in a (T-12) by a third person from team. Its role is to be a mediator in the discussion about disagreements in translation, and to write a report about two translations obtained.

- Translation from the Serbian language to the original questionnaire (translated back). This step is implemented by two translators whose native language is the language of the original questionnaire and who are not familiar with the concept of research.

- Working with T-12 version of the translation of the questionnaire, without the original scale - the questionnaire was translated into the original language. This is a valid checking process in order to gain assurance that the translated questionnaire is equal to the original.

- Review of the expert team, a comparison of all versions, control of language errors and entering a correction. In this phase, the composition of the expert team is crucial for achieving equivalence cross-cultural translated instruments. The professional team consisted: methodologist, clinician, lector, translators (who participated in translation) and the person who carried out the synthesis of the translation. The results was summated and reported to author. Author was verified whether the recommended methodology of the process of cultural adaptation escorted and whether they are in accordance with each step.

- Meeting of all the authors in order to conduct content validation (assessment of fitting each question in the questionnaire concept) - the final version of the questionnaire was organized and formed. During the validation of content the following aspects of the questionnaire was considered: the precision and clarity of the questions, whether the questions referred to the wrong answer, whether is necessary knowledge of the questionnaire examining theme by respondents. In this step were considered cancellation of the questions that do not correspond to the above aspects.

- After the translation and cultural adaptation, SMBQ questionnaire was placed on an electronic platform, together with the MBI questionnaire that was used to validate SMBQ questionnaire.

\section{Statistical data analysis}

Following metric characteristics were calculated: $\alpha$ - reliability coefficient Cronbach and associates; $\beta$ - the reliability of the first principal component; $\psi 1$ - representativeness; h1 - the homogeneity (the average correlation of items of the scale), extent of curvature, horizontal (Skewness) and vertical (Kurtosis). Correlations between subscales were examined. Latent structure SMBQ (maximumlikelihood extraction) was examined. The method of parallel analysis was used to select a number of factors. For calculation number of latent factors the Hull method was applied. Concurrent validity was examined by correlating the results of the questionnaire SMBQ and MBI. To confirm a one factor solution factor analysis was used.

\section{The assessment of metric characteristics of the questionnaire}

In research conducted with the assistance of instruments such as questionnaires, the first question is whether a 
questionnaire measure what it is supposed to measure. Questionnaires should ensure that in the measurements, more researchers interpret the results as close as possible, as well as that after multiple measurements again obtain similar results. ${ }^{14}$

Questionnaires whose metric properties was tested and in which was shown that the final results reflect the real situation, can be characterized as a "standardized". The questionnaire should meet the following requirements: validity, reliability, enough sensitivity to detect changes, and repeatability (the results are repeatable on the same population of respondents)..$^{12-14}$

Minimum requirements for the use of questionnaires are validity and reliability. ${ }^{13}$ The research results, obtained using a standardized questionnaire, may be accepted as relevant and reliable. ${ }^{14}$

Reliability and validity of the questionnaire are interrelated. Reliability is positively related to validity. With increasing reliability of the questionnaire, increases the validity. ${ }^{10-14}$

Consideration of the reliability of the questionnaire involves determining whether the questionnaire can get consistent and repeatable results. ${ }^{10-13}$

Consistency of results is assessed by considering whether all the questions in the questionnaire measures the same concept. Internal consistency of the questionnaire is to measure the link between the issues that constitute the questionnaire. To express the internal consistency is used Cronbach's alpha coefficient ( $\alpha$ cronbach). This size is also was explained by the observed variance of the score and the variance of each issue, and therefore represents a form of determining reliability. Cronbach alpha is a function of the correlation between the questions and the number of questions in the questionnaire. ${ }^{14}$ If there is no correlation between the questions, the total variance will be equal to the sum of variances of each questions, so that the cronbach will be 0 . In contrast, when all questions correlated, $\alpha$ cronbach will be 1 . When $\alpha$ cronbach above 0.7 consistency is is acceptable, when $\alpha$ Cronbach above 0.8 consistency is is good, and when is $\alpha$ cronbach above 0.9 consistency is excellent. ${ }^{14}$ Reliability also can be described by using the results of the repeatability and stability of the measurement. Repeatability of results is based on the analysis of the correlation between repeated measurements, which can be performed: after a specified time (reliability testing retesting) by two subjects (between reliability estimator) or the use of a number of similar forms (reliability of the equivalent forms). ${ }^{12-14}$

Reliability of the equivalent forms is examined by calculating the correlation between the results. ${ }^{14}$
Structural validity examines whether the questionnaire measure a concept that should be measured.

Structural validity includes:

1. Dimensionality of the questionnaire - examine whether the questions that belong to a domain is in relation to this domain.

2. The homogeneity of the questionnaire - whether the questions from one domain to reflect that domain.

3. Overlapping domains - examine whether the questions in greater correlation with the domain to which they belong, as compared to the correlation to other domains. ${ }^{14}$

Convergent validity examines whether the domains of the questionnaire, which in theory is considered to be related, interrelated. If it is proved that the two domains are correlated and if measure similar concept, they can be merger in one. ${ }^{14}$

Factor analysis is one of the most common multivariate statistical methods for creating and reviewing the structure of the questionnaire which are in the field of psychology. ${ }^{15}$ Factor analysis is used to detect relationships among component parts of the questionnaire or to reduce the number of questions in the questionnaire. Factor analysis can examine correlations between the questions in the questionnaire (exploratory factor analysis - EFA) or the fit of the hypothetical model questionnaire (confirmatory factor analysis - CFA). Accordingly, this assay has a very important role in the validation and testing the reliability of questionnaire structure. ${ }^{14}$

Questionnaires can consist one or more domains (also called latent variables or factors), with the aim of all the domains are directed towards one whole questionnaire, and measures in this totality. Domains are the thematic areas on which the questions grouped. Domains are immeasurable variables.

Exploratory factor analysis is a statistical technique that examines the correlation matrix. On the basis of the correlation can be identified questions that have a high correlation with each other, and that based on this combined into a single domain. If the model questionnaire already been checked, if its structure is already tested, confirmatory factor analysis has the advantage over exploratory. ${ }^{14}$

In order to reach a model in which the variance domain as lower Principal Components Analysis - PCA might be applied. This analysis includes the rotation of the domain, which leads to the reduction of dimensionality of the most suitable and detecting the model with a smaller number of domains. ${ }^{16,17}$ Frequently used in studies of the rotation varimax which aims to maximize 
the squared variance values in the factor loading to each of the questions, thus minimizing the number of high values of loading factor between questions. Varimax rotation belongs to the group of orthogonal rotation. ${ }^{14}$ We chosee this approach, becouse, when if we do not evaluate the validity or reliability of questionnaire, it could affect the believability of study's overall results. Results will be more accurate if the measures in a study are as reliable and valid as possible. Validity and reliability are not always aligned. Reliability is needed, but not sufficient to establish validity. We can get high reliability and low validity. This would happen when the wrong questions are asked over and over again, consistently yielding bad information. Also, if the results show large variation, they may be valid, but not reliable. For this reason, the method of factor analysis was used. Optimal implementation of the method of parallel analysis was performed. As a final confirmation has been carried out analysis of variance (ANOVA).

\section{RESULTS}

All licensed pharmacists have received an email by the Pharmaceutical Chamber of Serbia, with a link for accessing the questionnaires. The questionnaires were completed by 514 pharmacists.

First, the translation and cultural adaptation of the SMBQ was made. An expert team was formed consisting of two translators whose native language is Serbian and who are fluent in English, two translators whose native language is English and who are fluent in Serbian, a pharmacist employed in a pharmacy, a psychologist, a lector and a person who performed synthesis of translation. During this process, the team relied on the original - source questionnaire.

Translation and cultural adaptation of the questionnaire was carried out according to the ISPOR guidelines. At the beginning of the study, for every questionnaire obtained the license to use. The next step was the translation of "advance". Two translators have independently translated the questionnaire SMBQ from English to Serbian. A unique version of the questionnaire was created after comparing all three versions of translation. "Back translation" was undertaken by two independent translators whose native language is English, and who are not familiar with the concept of research. After that, have undertaken a comparison of all the translated version and made the necessary corrections in the questionnaire. The harmonization of all translated versions and review by the expert team, comparison of all versions, correction, and control of linguistic errors and the formation of a version for the prevarication study were undertaken.
Prevarication study, where the questionnaire was aimed at verifying interpretation and cognitive understanding, was tested for 30 pharmacists employed in Pharmacies in Serbia.

Respondents' comments on the structure of the questionnaire, the clarity of the issue and their understanding, were structured to be considered in the next stages of the cultural adaptation of the questionnaire.

After that, a meeting of the expert team was organized. Content validation was conducted (assessment of the integration of each question into the questionnaire concept) and the final version of the questionnaire was created. During the validation of the content, the following aspects of the questionnaire were considered: the precision and clarity of the question, whether the questions indicate the wrong answer and whether it is necessary for respondents to have knowledge of the topic questioned by the questionnaire. In this step, it was considered whether there are issues that for some reason need to be removed from the questionnaire, and the issues about which there was a dilemma at any stage of the cultural adaptation of the questionnaire were considered. For 16 items a consensus was reached between the members of the expert team, and for 6 items there were dilemmas. Items from the questionnaires that were discussed and about which there were dilemmas are shown in Table 1. For the sentence - I feel full of vitality, it was decided that the translation reads - I feel vital. Other proposals were rejected. In accordance with the concept of research and the cultural aspect, this formulation reflects the essence of the claim.

For the sentence - I have no energy for going to work in the morning - it was decided that the translation reads - I have no energy for going to work, because pharmacists in Serbian pharmacies are working in shifts, so it's not just about going to work in the morning. Lack of energy throughout the day may also indicate a risk of burning.

The most dilemma was around the item - My mind is cloudy. Several versions of the translation have been suggested here. It was decided to stay - I feel confused. Other versions of the translation are not in the spirit of the Serbian language, and can confuse the respondents. All items from the original questionnaire are accepted.

Psychometric characteristics of the scale and calculating correlation between variables was examined.

Metric characteristics were calculated using rtt10g. ${ }^{18}$

The following metric characteristics were calculated: $\alpha$ - reliability coefficient Cronbach and associates; $\beta$ - the reliability of the first principal component; $\psi 1$ - repre- 
sentativeness of the rocks; h1 - the homogeneity of the scale (the average correlation of items of the scale).

Metric characteristics of the SMBQ scale are shown in Table 2.

Really important or relevant findings from table 2 are summarized:

Cronbach's alpha is a measure of internal consistency, that is, how closely related a set of items are as a group. Cronbach's alpha is not a statistical test - it is a coefficient of reliability (or consistency). It is considered to be a measure of scale reliability. A "high" value for alpha does not imply that the measure is unidimensional. There are high Cronbach's alpha coefficients between the sub-scales. The resulting $\alpha$ coefficient of reliability ranges from 0 to 1 in providing this overall assessment of a measure's reliability. If all of the scale items are entirely independent from one another (i.e., are not correlated or share no covariance), then $\alpha=0$; and, if all of the items have high covariance's, then $\alpha$ will approach 1 as the number of items in the scale approaches infinity. $\beta$ coefitients reliability of the first main component are high. In other words, the higher the $\beta$ coefficient, the more the items have shared covariance and probably measure the same underlying concept.Many statistical tests and procedures are based on specific distributional assumptions. The assumption of normality is particularly common in classical statistical tests. Much reliability modeling is based on the assumption that the data follow a normal distribution. For this reason, they calculate the extent of curvature, horizontal (Skewness) and vertical (Kurtosis). Kolmogorov-Smirnov test provides a statistically significant deviation from the normal distribution. In all measures Kolmogorov-Smirnov statistics is significant at $\mathrm{p}<.01$ (that is not the normal distribution as expected on a sample of this size).

Correlations between variables (subscales) within the SMBQ scale are shown in Table 3.

High correlation coefitient means that a subscale score is a good estimate of scale score, and poor means that a subscale score might be dramatically discrepant from scalescore. Since the correlations between the subcalculum are significant, it means that the subcalcles measure the same parameter that measures the scale. This is also a confirmation of reliability.

Latent structure SMBQ scale was tested with maximumlikelihud extraction.

Eigenvalues latent factors SMBQ scale are shown in the Table 4.

As table 4 shown, a factor extraction method produces parameter estimates that are most likely to have produced the observed correlation matrix if the sample is from a multivariate normal distribution. The correlations are weighted by the inverse of the uniqueness of the variables, and an iterative algorithm is employed. When looking at the inherent value of the factors, it seems clear that one main component is isolated from the data. Eventually little switching takes place (which has an eigenvalue value greater than 1). Therefore, data can be optimally explained with one, at most two latent factors. To select a number of factors, method of the parallel analysis was used. There are the two forms of parallel analyzes, original and modified. ${ }^{19}$

Parallel analysis (PA) based on principal component analysis is shown in Table 5.

The classical method says that two factors explain the variance over randomness. The third extracted factor explains less variances than randomly generated.

Two-factor pattern matrix solutions are shown in Table 6. Optimal implementation of the method of parallel analysis was performed with the following parameters: ${ }^{20,21}$ Implementation details:

Correlation matrices analized: Pearson correlation matrices

Number of random correlation matrices: 500

Based on the matrix of the two-factor solution, we can also see that the scale can be explained by one, possibly 2 factors.

Parallel anallysis (PA) based on minimum rank factor anallysis are shown in Table 7.

This factor extraction method used to form uncorrelated linear combinations of the observed variables and to obtain the initial factor solution. It can be used when a correlation matrix is singular the first component has maximum variance. Successive components explain progressively smaller portions of the variance and are all uncorrelated with each other. A key decision in this process is determining the number of factors to retain for a group of variables of interest. Optimal implementation shows that it is best to keep only one factor.

To be sure, we calculated the number of latent factors and using the Hull method. All methods of retention factors were calculated using FACTOR 9.3.1.22,23

Implementation details for Hull method:

Goodness-of-fit index: CFI (Comparative Fit Index).

Method for dimensions extraction: ML.

Hull method for selecting the number of common factors are shown in Table 8.

A common problem in exploratory factor analysis is how many factors need to be extracted from a particular data set. New method for selecting the number of major common factors is the Hull method, which aims to find a model with an optimal balance between model fit and 


\begin{tabular}{|c|c|c|}
\hline $\begin{array}{c}\text { Item in the English version of the } \\
\text { questionnaire }\end{array}$ & Suggestions in translation & Adopted translation \\
\hline I feel full of vitality & $\begin{array}{l}\text { I feel energetic } \\
\text { I feel full of life } \\
\text { I feel vital }\end{array}$ & I feel vital \\
\hline My "batteries" are "dead" & $\begin{array}{c}\text { My "batteries are worn out" } \\
\text { I'm on the brink of strength } \\
\text { I feel exhausted }\end{array}$ & My "batteries are worn out" \\
\hline I feel burned out & $\begin{array}{l}\text { I feel like burning } \\
\text { I feel burned out at work } \\
\text { I feel exhausted }\end{array}$ & I feel burned out at work \\
\hline $\begin{array}{l}\text { I have no energy for going to work in } \\
\text { the morning }\end{array}$ & $\begin{array}{l}\text { I have no energy for going to work } \\
\text { I have no energy to start a job in the morning }\end{array}$ & $\begin{array}{l}\text { I have no energy for going } \\
\text { to work }\end{array}$ \\
\hline I feel relaxed & $\begin{array}{l}\text { I feel relaxed } \\
\text { I feel I'm not tense }\end{array}$ & I feel relaxed \\
\hline My mind is cloudy & $\begin{array}{c}\text { I feel gloomy } \\
\text { I feel my thoughts are foggy } \\
\text { I'm not in the mood } \\
\text { I'm thinking confusingly } \\
\text { I feel my thoughts are unclear } \\
\text { I feel confused }\end{array}$ & I feel confused \\
\hline
\end{tabular}

\begin{tabular}{|c|c|c|c|c|c|c|c|}
\hline \multicolumn{7}{|c|}{ Table 2 Metric characteristics of the SMBQ scale } \\
\hline Subscales & $\boldsymbol{\alpha}$ & $\boldsymbol{\beta}$ & $\boldsymbol{\Psi}$ & $\mathbf{h 1}$ & Skewness & $\begin{array}{c}\text { Kurtosis } \\
\text { Kolmogorov- } \\
\text { Smirnov Z }\end{array}$ \\
\hline SMBQ Emotional exhaustion (EE) & 0.9404 & 0.9404 & 0.988 & 0.7978 & 0.095 & -1.012 & 2.422 \\
\hline SMBQ Physical fatigue (PF) & 0.897 & 0.8981 & 0.9787 & 0.6852 & 0.252 & -0.995 & 2.917 \\
\hline SMBQ Cognitive weariness (Cog) & 0.9508 & 0.9509 & 0.9949 & 0.763 & -0.458 & -0.762 & 2.594 \\
\hline SMBQ Tension (TE) & 0.9194 & 0.9213 & 0.9899 & 0.7404 & -0.176 & -0.629 & 2.379 \\
\hline SMBQ Listlessness (LIS) & 0.8401 & 0.8407 & 0.9276 & 0.5677 & 0.277 & -1.022 & 3.418 \\
\hline
\end{tabular}

\begin{tabular}{|c|c|c|c|c|c|}
\hline Subscales & $\begin{array}{l}\text { SMBQ Emotional } \\
\text { exhaustion (EE) }\end{array}$ & $\begin{array}{c}\text { SMBQ Physical } \\
\text { fatigue (PF) }\end{array}$ & $\begin{array}{l}\text { SMBQ Cognitive } \\
\text { weariness (Cog) }\end{array}$ & $\begin{array}{l}\text { SMBQ Tension } \\
\text { (TE) }\end{array}$ & $\begin{array}{c}\text { SMBQ } \\
\text { Listlessness } \\
\text { (LIS) }\end{array}$ \\
\hline SMBQ Emotional exhaustion (EE) & 1 & & & & \\
\hline SMBQ Physical fatigue (PF) & $.903^{* *}$ & 1 & & & \\
\hline SMBQ Cognitive weariness (Cog) & $.759^{\star *}$ & $.745^{\star *}$ & 1 & & \\
\hline SMBQ Tension (TE) & $.883^{\star *}$ & $.825^{\star *}$ & $.775^{\star *}$ & 1 & \\
\hline SMBQ Listlessness (LIS) & $.850^{\star *}$ & $.865^{\star *}$ & $.697^{* *}$ & $.819^{* *}$ & 1 \\
\hline
\end{tabular}

number of parameters. The Hull method, however, suggested retaining the number of factors that the theoretical background to the inventory actually proposes. And this method suggest of keeping one factor, too. In this sense, the matrix of the structure of one-factor solutions is given. All questionnaires are in high correlation with the questionnaire itself. Therefore, the one-factor solutions is optimal.

The one-factor solutions matrix is shown in Table 9.
Correlations between SMBQ and MBI scales are shown in Table 10. Correlations between 2 scales are also high. This suggests that the two scales measure the same phenomenon.

The results show that the optimal one-factor solution. As a final confirmation has been carried out analysis of variance (ANOVA). ANOVA has shown that on the basis of the questionnaire is reasonable to analyze only one object being measured, and this is the main 


\begin{tabular}{|c|c|c|c|c|c|c|c|}
\hline \multirow[t]{2}{*}{ Factor } & \multicolumn{3}{|c|}{ Initial Eigenvalues } & \multicolumn{3}{|c|}{ Extraction Sums of Squared Loadings } & \multirow{2}{*}{$\begin{array}{c}\text { Rotation Sums of } \\
\text { Squared Loadings } \\
\text { Total }\end{array}$} \\
\hline & Total & $\%$ of Variance & Cumulative \% & Total & $\%$ of Variance & Cumulative \% & \\
\hline 1 & 14.467 & 65.757 & 65.757 & 14.143 & 64.288 & 64.288 & 13.437 \\
\hline 2 & 1.634 & 7.428 & 73.186 & 1.387 & 6.304 & 70.592 & 11.424 \\
\hline 3 & .902 & 4.098 & 77.284 & & & & \\
\hline 4 & .671 & 3.049 & 80.333 & & & & \\
\hline 5 & .532 & 2.418 & 82.751 & & & & \\
\hline 6 & .404 & 1.838 & 84.589 & & & & \\
\hline 7 & .380 & 1.729 & 86.319 & & & & \\
\hline 8 & .353 & 1.606 & 87.925 & & & & \\
\hline 9 & .342 & 1.555 & 89.480 & & & & \\
\hline 10 & .287 & 1.304 & 90.784 & & & & \\
\hline 11 & .267 & 1.212 & 91.996 & & & & \\
\hline 12 & .247 & 1.121 & 93.117 & & & & \\
\hline 13 & .209 & .949 & 94.066 & & & & \\
\hline 14 & .203 & .923 & 94.989 & & & & \\
\hline 15 & .187 & .852 & 95.842 & & & & \\
\hline 16 & .169 & .768 & 96.610 & & & & \\
\hline 17 & .159 & .723 & 97.332 & & & & \\
\hline 18 & .146 & .666 & 97.998 & & & & \\
\hline 19 & .139 & .631 & 98.629 & & & & \\
\hline 20 & .115 & .522 & 99.151 & & & & \\
\hline 21 & .100 & .454 & 99.605 & & & & \\
\hline 22 & .087 & .395 & 100.000 & & & & \\
\hline
\end{tabular}

Extraction Method: Maximum Likelihood. a. When factors are correlated, sums of squared loadings cannot be added to obtain a total variance.

\begin{tabular}{|c|c|c|c|}
\hline \multicolumn{4}{|c|}{ Table 5: Parallel analysis (PA) based on principal component analysis } \\
\hline Variable & $\begin{array}{c}\text { Real-data } \\
\text { eigenvalues }\end{array}$ & $\begin{array}{c}\text { Mean of random } \\
\text { eigenvalues }\end{array}$ & $\begin{array}{c}\text { 95 percentile of random } \\
\text { eigenvalues }\end{array}$ \\
\hline 1 & $14.46660^{*}$ & 1.38805 & 1.45103 \\
\hline 2 & $1.63424^{*}$ & 1.32322 & 1.36652 \\
\hline 3 & 0.90160 & 1.27422 & 1.31108 \\
\hline
\end{tabular}

* Advised number of dimensions: 2

factor that is calculated by using the extracted factors of maximum-likelihood result $(\mathrm{F}(3.510)=376.112$, $\mathrm{p}=0.01)$. A higher score on this variable indicates a higher burnout. He was taken as a criterion in the regression variables and MBI as a predictor.

The results are shown in Table 11.

A large percentage of the variance of the general factors is illustrated by way of MBI scale (69\%). All three were significant predictors. Zero-order correlations were again very high. This means that these two questionnaires have a similar structure.

The noticed levels of burnout in the pharmacist population estimated by the SMBQ scale are shown in the table 12.
The values obtained in this study indicate the presence of a moderate risk of burning in all pharmacists examined. The results of the burnout risk assessment show that there is no risk of burnout in the majority of examined pharmacists $(81.9 \%)$ and that only $12.3 \%$ of the pharmacists are at high risk of burnout.

\section{DISCUSSION}

Based on the set goals, it is possible to summarize the really important or relevant findings:

1. Shirom-Melamed Burnout Questionnaire as the instriment for the assess burnout syndrome at 


\begin{tabular}{|c|c|c|}
\hline \multicolumn{3}{|c|}{ Pattern Matrix ${ }^{a}$} \\
\hline & \multicolumn{2}{|c|}{ Factor } \\
\hline & 1 & 2 \\
\hline I feel burned out (EE) & .934 & -.057 \\
\hline I feel alert (LIS) & .866 & -.067 \\
\hline My "batteries" are "dead" (EE) & .863 & .037 \\
\hline I feel refreshed (PF) & .853 & -.125 \\
\hline I feel tired (PF) & .823 & .031 \\
\hline I feel tense (TE) & .799 & .130 \\
\hline I feel relaxed (TE) & .787 & -.087 \\
\hline I feel fed up (EE) & .784 & .113 \\
\hline I feel full of vitality (LIS) & .777 & -.052 \\
\hline I feel physically exhausted (PF) & .751 & .106 \\
\hline I feel mentally fatigued (EE) & .745 & .195 \\
\hline I feel restless (TE) & .678 & .244 \\
\hline I feel sleepy (LIS) & .644 & .178 \\
\hline I have no energy for going to work in the morning (PF) & .633 & .231 \\
\hline I feel intense inner tension (TE) & .549 & .375 \\
\hline I feel active (LIS) & .510 & .103 \\
\hline I have difficulty thinking about complex things (Cog) & -.173 & 1.051 \\
\hline My mind is cloudy (Cog) & -.042 & .913 \\
\hline My thinking process is slow (Cog) & -.045 & .908 \\
\hline I have difficulty concentrating (Cog) & .085 & .822 \\
\hline I feel I am disorganized lately (Cog) & .119 & .736 \\
\hline I am too tired to think clearly (Cog) & .323 & 620 \\
\hline
\end{tabular}

Extraction Method: Maximum Likelihood.

Rotation Method: Promax with Kaiser Normalization.

a. Rotation converged in 3 iterations.

\begin{tabular}{|c|c|c|c|}
\hline \multicolumn{3}{|c|}{ Table 7: Parallel anallysis (PA) based on minimum rank factor anallysis } \\
\hline Variable & $\begin{array}{c}\text { Real-data } \\
\text { \% of variance }\end{array}$ & $\begin{array}{c}\text { Mean of random } \\
\% \text { of variance }\end{array}$ & $\begin{array}{c}\text { 95 percentile of random } \\
\text { \% of variance }\end{array}$ \\
\hline 1 & $68.7^{*}$ & 9.1 & 10.2 \\
\hline 2 & 7.4 & 8.5 & 9.4 \\
\hline
\end{tabular}

* Advised number of dimensions:1

\begin{tabular}{|c|c|c|c|}
\hline \multicolumn{4}{|c|}{ Table 8: Hull method for selecting the number of common factors } \\
\hline Number of factors & Goodnes - of -fit values & Degrees of freedom & Scree test values \\
\hline 0 & 0.000 & 22 & 0.000 \\
\hline 1 & 0.802 & 44 & $7.564^{*}$ \\
\hline 2 & 0.903 & 65 & 2.725 \\
\hline 3 & 0.939 & 85 & 0.000 \\
\hline
\end{tabular}

* Advised number of common factors: 1 


\begin{tabular}{|c|c|}
\hline \multicolumn{2}{|l|}{ Factor Matrix ${ }^{a}$} \\
\hline & Factor \\
\hline & 1 \\
\hline I feel mentally fatigued (EE) & .899 \\
\hline I feel tense (TE) & .898 \\
\hline I feel restless (TE) & .881 \\
\hline My "batteries" are "dead" (EE) & .876 \\
\hline I feel intense inner tension (TE) & .869 \\
\hline I feel fed up (EE) & .865 \\
\hline I feel burned out (EE) & .860 \\
\hline I am too tired to think clearly (Cog) & .855 \\
\hline I feel tired (PF) & .828 \\
\hline I feel physically exhausted (PF) & .824 \\
\hline I have no energy for going to work in the morning (PF) & .821 \\
\hline I have difficulty concentrating (Cog) & .793 \\
\hline I feel alert (LIS) & .787 \\
\hline I feel sleepy (LIS) & .784 \\
\hline I feel I am disorganized lately (Cog) & .753 \\
\hline My mind is cloudy (Cog) & .747 \\
\hline My thinking process is slow (Cog) & .739 \\
\hline I have difficulty thinking about complex things (Cog) & .736 \\
\hline I feel refreshed (PF) & .721 \\
\hline I feel full of vitality (LIS) & .713 \\
\hline I feel relaxed (TE) & .694 \\
\hline I feel active (LIS) & .587 \\
\hline
\end{tabular}

Extraction Method: Maximum Likelihood.

a. 1 factors extracted. 5 iterations required.

Table 10: Correlations between SMBQ and MBI scales

\begin{tabular}{|c|c|c|c|}
\hline \multicolumn{4}{|c|}{ Table 10: Correlations between SMBQ and MBI scales } \\
\hline & $\begin{array}{c}\text { MBI } \\
\text { Exhaustion }\end{array}$ & $\begin{array}{c}\text { MBI } \\
\text { Depersonalization }\end{array}$ & MBI Personal achievement \\
\hline SMBQ Emotional exhaustion (EE) & $.805^{\star *}$ & $.805^{* *}$ & $-.761^{\star *}$ \\
\hline SMBQ Physical fatigue (PF) & $.908^{\star *}$ & $.907^{* *}$ & $-.850^{* *}$ \\
\hline SMBQ Cognitive weariness (Cog) & $.652^{\star *}$ & $.647^{* *}$ & $-.593^{* *}$ \\
\hline SMBQ Tension (TE) & $.722^{* *}$ & $.713^{* *}$ & $-.669^{* *}$ \\
\hline SMBQ Listlessness (LIS) & $.766^{* *}$ & $.767^{* *}$ & $-.719^{* *}$ \\
\hline
\end{tabular}

$* *-p<.01$

\begin{tabular}{|c|c|c|c|c|c|c|c|c|c|}
\hline \multirow{2}{*}{\multicolumn{2}{|c|}{$\begin{array}{l}\text { Model } \\
\text { B }\end{array}$}} & \multicolumn{2}{|c|}{$\begin{array}{l}\text { Unstandardized } \\
\text { Coefficients }\end{array}$} & \multirow[t]{2}{*}{$\begin{array}{c}\text { Standardized } \\
\text { Coefficients }\end{array}$} & \multirow[t]{2}{*}{$\mathbf{t}$} & \multirow{2}{*}{$\begin{array}{l}\text { Sig. } \\
\text { Zero- } \\
\text { order }\end{array}$} & \multicolumn{3}{|c|}{ Correlations } \\
\hline & & Std. Error & Beta & & & & Partial & Part & \\
\hline \multirow[t]{4}{*}{1} & (Constant) & -2.214 & .394 & & -5.623 & .000 & & & \\
\hline & MBI_Exhaustion & .298 & .102 & .517 & 2.919 & .004 & .827 & .128 & .072 \\
\hline & MBI_Depersonalization & .317 & .127 & .530 & 2.501 & .013 & .823 & .110 & .062 \\
\hline & MBI_Personal achievement & .169 & .068 & .227 & 2.479 & .013 & -.771 & .109 & .061 \\
\hline
\end{tabular}

a. Dependent Variable: SMBQ_Burnout 
Table 12: Burnout levels in the pharmacist population estimated by SMBQ scale

\begin{tabular}{|c|c|c|}
\hline Burnout levels & $\mathbf{N}$ & $\%$ \\
\hline No risk of burnout & 421 & 81.9 \\
\hline Moderate risk of burout & 30 & 5.8 \\
\hline High risk of burnout & 63 & 12.3 \\
\hline Total & 514 & 100.0 \\
\hline
\end{tabular}

work, showed satisfactory validity on a sample of pharmacists at the primary level of health care.

2. We can be sure that the single-factor solution is optimal for several reasons:

- Two retention methods have recommended a single-factor solution;

- Correlation between two factors from a two-factor solution is too high for them to be retained as independent entities;

- In the matrix of the single-factor solution, there is no single variable having a saturation of less than 0.30 , and even close to that value that can suggest that the variable does not belong to the factor.

- Finally, the one-factor solution is completely logical when we look at the high correlations between all five questionnaires, calculated using the original scoring method.

3. The investigated pharmacists in the sample were showed a risk of burnout at work.

Reliability SMBQ is high, with high correlations between the items themselves, as well as the correlation between the scales themselves.

Correlations between variables (subscales) within the SMBQ scale are high, too. This is shown by research conducted with this scale..$^{8-24-26}$

In this way, verified the impact of each question on the questionnaire consistency.

After examining the eigenvalues of factors seems clear from the data that isolates each major component. Eventually bit shifting one (which has an inherent value greater than 1). So data can be explained by the optimal one, at most two latent factors.

A good rule of thumb for determining the number of factors is to only choose factors with associated eigenvalue (or variance) greater than $1 .{ }^{23}$ In most analyses, only the first few components account for meaningful amounts of variance, so only these first few components are retained, interpreted, and used in subsequent analyses (such as in multiple regression analyses). In this research the number of components extracted in a principal component analysis is equal to the number of observed variables being analyzed. This is often in situations where questionnaires measure similar phenomenon. $^{27}$

Classical methods shows that two factors explain the variance over accidents. For the third extracted factor explains less variance than randomly generated. ${ }^{28}$

For example, research conducted in analysis of the 7-item job satisfaction questionnaire (when is situation with SMBQ), it is likely that only the first two components would account for a meaningful amount of variance; therefore only these would be retained for interpretation. ${ }^{29}$

Reviewing two factor solutions, it can be seen clearly that the first factor I fall all items of cognitive fatigue, which saturate the items were another factor. So, the only thing that distinguishes the cognitive component. However, the correlation between these two factors is very high $(\mathrm{r}=.75, \mathrm{p}<.001)$, thus demonstrating that it is probably still-factor solution is optimal. Research indicated that the tension set of SMBQ items were the problem, as well as local dependency throughout the scale. The most widely used instrument is the Maslach Burnout Inventory (MBI) and the conceptual basis for $\mathrm{MBI}$ is thus often considered as synonymous with the construct burnout.

Given the overlapping domains, there is a high possibility that both the scale measures the same domain. This is also shown a high probability that in the present SMBQ one factor solution. ${ }^{8}$

The values obtained in this study indicate the presence of a moderate risk of burning in all pharmacists examined. The results of the burnout risk assessment show that there is no risk of burnout in the majority of examined pharmacists $(81.9 \%)$ and that only $12.3 \%$ of the pharmacists are at high risk of burnout.

These are significantly different results compared to the survey conducted in 2012 on a sample of 647 pharmacies employed in pharmacies in Serbia, where $44.4 \%$ of pharmacists showed a high risk at work burnout. ${ }^{2}$ Also, a survey on a sample of 386 pharmacists in Serbia conducted in 2013 showed that $42 \%$ of pharmacists were at high risk of burnout. In these studies, Freudenberg scale for measuring burnout syndrome was used..$^{30}$ 
This difference in results can be interpreted by a different methodology of research, as well as changes in the pharmacy practice that occurred in the previous period. However, burnout studies in different populations show different degrees of burnout risk. ${ }^{31-33}$

\section{CONCLUSION}

The best way to test concurrent validity is to compare the results of two specific questionnaires. For comparison was used MBI. Concurrent validity was examined by correlating the results of the questionnaire SMBQ and MBI. Since each question reflects one domain, only tested the correlation between the final results of the questionnaire. Research has shown high reliability and validity SMBQ. Psychometric attributes are supported by the original development papers.

Statistical analysis includes an analysis of characteristics of the fundamental questions, checking the psychometric characteristics of the questionnaire and the questionnaire structure testing using factor analysis.

Research has shown that we can be sure that the one-factor solution for SMBQ scale is optimal, and SMBQ scale have shown high reliability and validity. SMBQ scale is suitable instrument to measure job burnout at pharmacist. The scale showed the one - factor structure at the pharmacist population. Differences in findings can be explained by the fact that respondents involved in this research, unlike others, have approximately the same level of education, so they can be expected to have equal expectations and responsibilities in relation to what they want to achieve.

It would be useful to extend assess the structure of the SMBQ scale at a different type of population in order to confirm or disprove a one-factor solution.

The values obtained in this study indicate the presence of a moderate risk of burning in all pharmacists examined. The validated instrument will be used for the purpose of testing burnout on the population of pharmacists as part of the study of the examine impact of burnout on professional and personal competencies. Further research is needed to provide to more precise evidence individually for factors that may affect burnout.

Pharmaceutical profession as a part of the medical profession, due to its responsibility for human life, is especially dedicated to preserving quality and setting high standards in work. In order to achieve this, it is essential that pharmacists have appropriate competencies that enable them to make the right decisions in the best interests of patients. On the other hand, due to the nature of pharmacist work in the modern conditions of pharmaceutical practice, many factors can contribute to increasing the burnout risk at work, which can affect the attitudes and beliefs that pharmacists may have about their own work with patients, and therefore on professional and personal competencies.

Given the large number of factors that can influence burnout, but also to demonstrate competencies in everyday work, it is necessary to continue with the conduct of similar research in order to achieve as precise findings as possible.

\section{ACKNOWLEDGEMENT}

We would like to thank all the study participants for their contribution to this study. We are also grateful to the Pharmaceutical Chamber of Serbia for providing demografic data for the survey and help in distributing the questionnaires.

The work of DK was supported by the Ministry of Education, Science and Technological Development of Republic of Serbia, Project No. 41004.

\section{CONFLICT OF INTEREST}

The authors declare no conflict of interest.

\section{ABBREVIATION USED}

SMBQ: Shirom-Melamed Burnout Questionnaire; MBI: Maslach Burnout Inventory; ISPOR: International Society for Pharmacoeconomics and Outcomes Research; EFA: Exploratory factor analysis; CFA: Confirmatory factor analysis; PCA: Principal Components Analysis; EE: Emotional exhaustion; PF: Physical fatigue; Cog: Cognitive weariness; TE: Tension; LIS: Listlessness; PA: Parallel analysis; CFI: Comparative Fit Index; ANOVA: Analysis of variance.

\section{REFERENCES}

1. Jocić $D$, Krajnović $D$. The influence of autogenic training on state anxiety reduction among community pharmacists in Serbia. HealthMED Journal 2012;6(8):2675-85.

2. Jocić D, Krajnović D. State Anxiety, Stress and Burnout Syndrome Among Community Pharmacists: Relation With Pharmacists' Attitudes and Beliefs. Indian Journal of Pharmaceutical Education and Research 2014;48(2):9-15.

3. Melamed S, Shirom A, Toker S, Berliner SShapira: Burnout and risk of cardiovascular disease: evidence, possible causal paths, and promising research directions. Psychol Bull 2006;132(3):327-53.

4. Leiter MP, Bakker AB, Maslach C. Burnout at Work: A psychological perspective, Hove, Sussex: Psychology Press, 2014.

5. Nazari H, Jariani M, Beiranvand S, Saki M, Aghajeri N, Ebrahimzadeh F. The Prevalence of Job Stress and its Relationship with Burnout Syndrome among the Academic Members of Lorestan University of Medical Sciences. Journal of Caring Sciences 2016;5(1):75-84.

6. Fradelos E, Tzitzikos G, Giannouli V, Argyrou P, Vassilopoulou C, Theofilou P. Assessment of burn-out and quality of life in nursing professionals: the contribution of perceived social support. Health Psychology Research 2014;2:984. 
7. Maslach C, Jackson S. Banishing Burnout, Jossey-Bass, San Francisco, USA, 2003.

8. Lundgren -Nilsson A, Jonsdottir IH, Pallant J, Ahlborg G. Internal construct validity of the Shirom-Melamed Burnout Questionnaire (SMBQ). BMC Public Health. 2012;12:1.

9. Acquardro C. ERIQA recommendations for translation and cultural adaptation of HRQL measures. ISPOR 6th Annual European Congress; 2003 November 9-11; Barcelona Spain; 2003.

10. Wild D, Grove A, Martin M, Eremenco S, McElroy S, Verjee-Lorenz A, et al. Principles of good practice for the translation and cultural adaptation process for patient-reported outcomes (PRO) measures: Report of the ISPOR Task Force for Translation and Cultural Adaptation. Value Health 2005;8(2):94-104.

11. Dewolf L, Koller M, Velikova G, Johnson C, Scott N, Bottomley A. EORTC Quality of Life Group Translation Procedure. 2009 [cited 04.12.2011]; Available from: http://groups.eortc.be/qol/downloads/translation_manual_2009.pdf

12. Acquadro C, Conway K, Hareendran A, Aaronson N. Literature review of methods to translate health-related quality of life questionnaires for use in multinational clinical trials. Value Health 2008;11(3):509-21.

13. Costa F, Guggan C, Bates I. A systematic approach to cross-cultural adaptation of survey tools", Pharmacy Practice 2007; 5(3):115 -24.

14. Fayers PM, Machin D. Quality of life: Assessment, Analysis and Interpretation of patient reported outcomes. 2nd ed. Chichester: John Wiley \& Sons; 2007.

15. Norman GR, Streiner DL. PDQ Statistics. 3 ed. Ontario: BC Decker Inc; 2003.

16. Holland SM. Principal Components Analysis (PCA). 2008 [cited 28.06.2012]; Available from: http://strata.uga.edu/software/pdf/pcaTutorial.pdf

17. Kootstra GJ. Exploratory Factor Analysis: Theory and Application. 2004 [cited 28.06.2012]; Available from: http://www.let.rug.nl/ nerbonne/teach/remastatsmeth-Seminar/Factor-Analysis-Kootstra-04.PDF

18. Knežević GD, Momirović K. RTT9G i RTT10G - two programs for the analysis of metric characteristics of composite measuring instruments. In: Petar Kostic [ed.] Measurement in psychology, Institute of Criminological and Sociological Research, Belgrade, 1996:37-56.

19. Horn LJ. A rationale and test for the number of factors in factor analysis. Psychometrica 1965;30(2):179-85.

20. Timmerman ME, Lorenzo-Seva U. Dimensionality assessment of ordered polytomous items with Parallel Analysis. Psychological Methods, 2011;16(2):209-20.

\section{PICTORIAL ABSTRACT}

\section{Shirom-Melamed Burnout Questionnaire (SMBQ)}

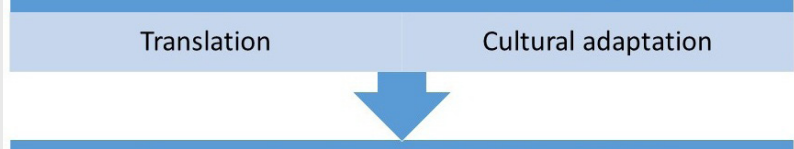

Assessment metric characteristics of SMBQ

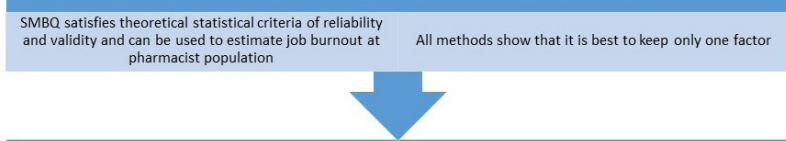

Degree of burnout in the pharmacist population
No risk of burnout $-81.9 \%$
Moderate risk of burnout $-5.8 \%$
High risk of burnout $-12.3 \%$

21. Buja A, Eyuboglu, N. Remarks on parallel analysis. Multivariate Behavioral Research.1992;27(4):509-40.

22. Lorenzo-Seva U, Ferrando PJ. Factor: a computer program to fit the exploratory factor analysis model. Behavior Research Methods, 2006;38(1):88-91.

23. Lorenzo-Seva U, Timmerman ME, Kiers HA. The Hull Method for Selecting the Number of Common Factors.Multivariate Behavioral Research 2011; 46(2):340-64.

24. Melamed S, Kushnir T, Shirom A. Burnout and risk factors for cardiovascular diseases. Behavioral Medicine 1992;18(2):53-60.

25. Armon G, Melamed S, Shirom A, Berliner ST, Shapira I. The associations of the five factor model of personality with inflammatory biomarkers: A four-year prospective study. Personality and Individual Differences, 2013;54(6):750-5.

26. Shirom A, Toker S, Melamed S, Berliner S, Shapira I. Life and job satisfaction as predictors of the incident diabetes. Applied Psychology: Health and WellBeing, 2012;4(1):31-48.

27. Kamil ML, Pearson PD, Moje EB, Afflerbach P. Handbook of Reading Research, Routledge, New York, 2011.

28. Kovacevic N, McIntosh R. Groupwise independent component decomposition of EEG data and partial least square analysis. Neurolmage 2007;35(3):1103-12.

29. Norris M, Lecavalier L. Evaluating the Use of Exploratory Factor Analysis in Developmental Disability Psychological Research. Journal of Autism and Developmental Disorders.2009;40(1):8-20.

30. Jocic D, Krajnovic D, Lakic D. Job Burnout Predictors In Community Pharmacists In Serbia, Value in Health, 2016;19(7):A475.

31. Sirsawy U, Steinberg WJ, Raubenheimer JE. Levels of burnout among registrars and medical officers working at Bloemfontein public healthcare facilities in 2013. South African Family Practice; 2016;58(6):213-18

32. Prendergast C, Ketteler E, Evans G. Burnout in the Plastic Surgeon: Implications and Interventions. Aesthetic Surgery Journal, 2017;37(3):363-68.

33. da Costa BRC, Pinto ICJF. Stress, Burnout and Coping in Health Professionals: A Literature Review. Journal of Psychology and Brain Studies, 2017; 1:1. Available in: http://www.imedpub.com/psychology-and-brainstudies/. Date of access: 01/20/2017.

\section{SUMMARY}

- Due to the nature of work in pharmacies pharmacists may occur job burnout, which can affect the quality of health services.

- It is necessary to use validated instruments to test the burnout. One type of instrument is ShiromMelamed Burnout Questionnaire (SMBQ).

- A cross-sectional survey design was used. Data were obtained from self-administered instrument from January 2017 to March 2017.

- The cultural adaptation of the SMBO questionnaire was made according to ISPOR guidelines. - To assess the burnout risk Shirom-Melamed Burnout Questionnaire (SMBQ) was used. For validation SMBQ Maslach Burnout Inventory (MBI) was used.

- Shirom-Melamed Burnout Questionnaire as the instriment for the assess burnout syndrome at work, showed satisfactory validity on a sample of pharmacists at the primary level of health care. is optimal.

We can be sure that the single-factor solution

- The investigated pharmacists in the sample were showed a risk of burnout at work. 


\section{About Authors}

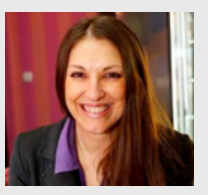

Dragana Jocic: Is a graduate psychologist and a graduate pharmacist at the University of Belgrade. She has employed in BENU, a company Phoenix Group, as Human Resources Director. She has also engaged at the Department of Social Pharmacy and Pharmaceutical Legislation, Faculty of Pharmacy, University of Belgrade. The main areas of her interest: Pharmaceutical practice, Behavioral and psychological aspects of pharmaceutical practice, Research and development of pharmaceutical practice, Human resources management, Training and development of employees. She completed Postgraduate Qualification Study -Specialization at the Faculty of Pharmacy, University of Belgrade. PhD student at the Faculty of Medical Sciences - University of Kragujevac, Department of Preventive Medicine. She is licensed educator of communication skills, assertive and autogenic training. Trained in psychotherapy direction - Transactional Analysis and Cognitive behavioral therapy. She published 14 papers, 5 in international peer-reviewed journals and 36 abstracts in national and international meetings. Co-author of the book "Communication in pharmacy practice."

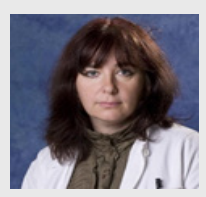

Nela Djonovic: Head of the Department of Doctoral Studies-Preventive Medicine, Head of the Department of Hygiene and Ecology, Head of the Department for Hygiene Specialization and narrow specialization in Dietotherapy. Faculty of Medicine (1992), M.Sc. (1995) and doctoral studies (1999) graduated from the Faculty of Medicine, University of Kragujevac. Since 1994, she has been employed at the Faculty of Medical Sciences, University of Kragujevac. At the pharmacy studies, he is the manager of the Nutritive Supplements course. Specialization in hygiene ended in 2002. at the Faculty of Medicine, University of Belgrade. She is also employed in the Institute of Public Health Kragujevac as assistant director for scientific research. She has published more than 70 papers out of which 22 in magazines from the $\mathrm{SCl}$ list.

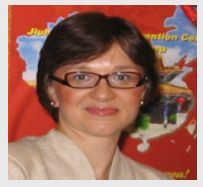

Dusanka Krajnovic: PhD, Head of the Department for Social Pharmacy and Pharmacy Legislation at the University of Belgrade -Faculty of Pharmacy. Dr Krajnovic holds presently an Associate Professor position at the Faculty of Pharmacy, University of Belgrade and is Fellow at the Center for the Study of Bioethics, General Secretary of the Serbian Unit of the UNESCO Chair in Bioethics. Dr. Krajnović has MA degree in Pharmaceutical Marketing and Management from the same Faculty and MA degree in Pharmaceutical Legislation and Pharmaceutical economy. She is a member of the Faculty of Pharmacy Ethics Committee. As guest speaker at various national and international conferences and presented more than 200 poster and oral presentations at national and international confernces. She has more than 20 original full-papers published in indexed scientific journals with impact factor and, amongst other work, she has been the supervisor of 1 Master and 4 PhD students. Her publications include various books and edited collections as well as numerous articles from the domain of social pharmacy and public health, (bio-pharmacy) ethics, decision making and pharmacy legislation.

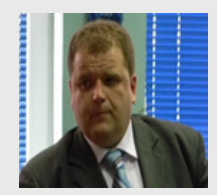

Srdjan Stefanovic: Assistant Professor of Clinical Pharmacy, Faculty of Medical Sciences, University of Kragujevac, M.D: 2001, Medical Faculty, University of Kragujevac, Serbia; Specialty: 2009, Medical Faculty, University of Kragujevac, Serbia, Clinical Pharmacology; PhD: 2010, Medical Faculty, University of Kragujevac, Serbia, Clinical and Experimental Pharmacology. External Expert for the Evaluation of Clinical Documentation for the Marketing Autorization in Serbia, Medicines and Medical Devices Agency of Serbia, Member of the Committee for Marketing Authorization of Human Medicinal Products, Medicines and Medical Devices Agency of Serbia.

Svetlana Stojkov: She completed specialist academic studies - Pharmacoeconomy and Pharmaceutical Legislation (2006) and Pharmaceutical Marketing and Management (2008) at the Faculty of Pharmacy, University of Belgrade. Magisterial thesis on the topic of Specificity of application of marketing in pharmacies, was defended in 2008 at the Faculty of Economics in Subotica, University of Novi Sad. Doctoral dissertation in the field of social pharmacy, entitled Development, application and evaluation of instruments for assessing the competence of pharmacists in community pharmacies in Serbia at the Faculty of Pharmacy, University of Belgrade, was defended in 2016 . Since May 2014 he has been the Director of the Pharmaceutical Chamber of Serbia.

Sanja Kocic: Associate Faculty of Medical Sciences, University of Kragujevac. The main area of her interest: Social medicine. She specializes in Social Medicine at the University of Medicine School of Medicine. She received her doctorate in $\mathbf{2 0 0 5}$ at the Faculty of Medicine, University of Kragujevac. She is also employed as a specialist in social medicine at the Institute for Health Protection in Kragujevac.

Cite this article: Jocic DD, Djonovic NZ, Krajnovic DM, Stefanovic SM, Stojkov SS, Kocic SS. Cultural Adaptation and Examination of Metric Characteristics Shirom-Melamed Burnout Questionnaire (Smbq) On a Sample of Pharmacists in Serbia. Indian J of Pharmaceutical Education and Research. 2018;52(1):166-80. 